\title{
Post-stroke dementia - a comprehensive review
}

Milija D. Mijajlović ${ }^{*}$, Aleksandra Pavlović ${ }^{1}$, Michael Brainin², Wolf-Dieter Heiss ${ }^{3}$, Terence J. Quinn ${ }^{4}$, Hege B. Ihle-Hansen ${ }^{5}$, Dirk M. Hermann ${ }^{6}$, Einor Ben Assayag ${ }^{7,8}$, Edo Richard ${ }^{9}$, Alexander Thiel ${ }^{10}$, Efrat Kliper ${ }^{7,8}$, Yong-II Shin ${ }^{11}$, Yun-Hee Kim ${ }^{12}$, SeongHye Choi ${ }^{13}$, San Jung ${ }^{14}$, Yeong-Bae Lee ${ }^{15}$, Osman Sinanović ${ }^{16}$, Deborah A. Levine ${ }^{17}$, Ilana Schlesinger ${ }^{18,19}$, Gillian Mead ${ }^{20}$, Vuk Milošević ${ }^{21}$, Didier Leys ${ }^{22}$, Guri Hagberg ${ }^{5}$, Marie Helene Ursin ${ }^{5}, Y_{v o n n e ~ T e u s c h l^{2}}$, Semyon Prokopenko ${ }^{23}$, Elena Mozheyko ${ }^{23}$, Anna Bezdenezhnykh ${ }^{23}$, Karl Matz ${ }^{2}$, Vuk Aleksić ${ }^{24}$, DafinFior Muresanu ${ }^{25}$, Amos D. Korczyn ${ }^{26}$ and Natan M. Bornstein ${ }^{7,8}$

\begin{abstract}
Post-stroke dementia (PSD) or post-stroke cognitive impairment (PSCI) may affect up to one third of stroke survivors. Various definitions of PSCI and PSD have been described. We propose PSD as a label for any dementia following stroke in temporal relation. Various tools are available to screen and assess cognition, with few PSD-specific instruments. Choice will depend on purpose of assessment, with differing instruments needed for brief screening (e.g., Montreal Cognitive Assessment) or diagnostic formulation (e.g., NINDS VCl battery). A comprehensive evaluation should include assessment of pre-stroke cognition (e.g., using Informant Questionnaire for Cognitive Decline in the Elderly), mood (e.g., using Hospital Anxiety and Depression Scale), and functional consequences of cognitive impairments (e.g., using modified Rankin Scale). A large number of biomarkers for PSD, including indicators for genetic polymorphisms, biomarkers in the cerebrospinal fluid and in the serum, inflammatory mediators, and peripheral microRNA profiles have been proposed. Currently, no specific biomarkers have been proven to robustly discriminate vulnerable patients ('at risk brains') from those with better prognosis or to discriminate Alzheimer's disease dementia from PSD. Further, neuroimaging is an important diagnostic tool in PSD. The role of computerized tomography is limited to demonstrating type and location of the underlying primary lesion and indicating atrophy and severe white matter changes. Magnetic resonance imaging is the key neuroimaging modality and has high sensitivity and specificity for detecting pathological changes, including small vessel disease. Advanced multi-modal imaging includes diffusion tensor imaging for fiber tracking, by which changes in networks can be detected. Quantitative imaging of cerebral blood flow and metabolism by positron emission tomography can differentiate between vascular dementia and degenerative dementia and show the interaction between vascular and metabolic changes. Additionally, inflammatory changes after ischemia in the brain can be detected, which may play a role together with amyloid deposition in the development of PSD. Prevention of PSD can be achieved by prevention of stroke. As treatment strategies to inhibit the development and mitigate the course of PSD, lowering of blood pressure, statins, neuroprotective drugs, and anti-inflammatory agents have all been studied without convincing evidence of efficacy. Lifestyle interventions, physical activity, and cognitive training have been recently tested, but large controlled trials are still missing.
\end{abstract}

Keywords: Cognitive impairment, Dementia, Definitions and classification, Diagnosis, Neuroimaging, Interventions, Biomarkers, Stroke

\footnotetext{
*Correspondence: milijamijajlovic@yahoo.com

${ }^{1}$ Neurology Clinic, Clinical Center of Serbia, School of Medicine, University of

Belgrade, Dr Subotica 6, 11000 Belgrade, Serbia

Full list of author information is available at the end of the article
}

(c) The Author(s). 2017 Open Access This article is distributed under the terms of the Creative Commons Attribution 4.0 International License (http://creativecommons.org/licenses/by/4.0/), which permits unrestricted use, distribution, and reproduction in any medium, provided you give appropriate credit to the original author(s) and the source, provide a link to the Creative Commons license, and indicate if changes were made. The Creative Commons Public Domain Dedication waiver (http://creativecommons.org/publicdomain/zero/1.0/) applies to the data made available in this article, unless otherwise stated. 


\section{Background}

Stroke is a leading cause of disability [1]. Research and interventions have historically focused on physical disabilities [2], while cognitive impairment - an important aspect for stroke survivors - has been rather neglected $[3,4]$. Even minor stroke affects daily functioning, executive functions, and cognition, consequently affecting participation, quality of life, and return to work [5]. Stroke survivors are at increased risk of developing cognitive impairment. Obviously, the acute tissue damage may affect cognition. Nevertheless, despite prospective data being available, results are conflicting and the direct cognitive effect of a stroke event beyond the cognitive decline associated with age and vascular risk factors remains poorly understood. Physical impairments tend to improve, to a greater or lesser degree, following stroke; however, for reasons which remain unknown, cognitive impairments progressively worsen.

This review paper is based on the proceedings of the International Congress on Vascular Dementia, Ljubljana, 2015, at which reviews of the literature on post-stroke cognitive impairment (PSCI) and post-stroke dementia (PSD) were presented by leaders of the field and discussed by a broad audience. Key thematic areas were chosen for further elucidation in smaller working groups and, after further discussions, a final review was compiled.

\section{Methods of research}

Using a focused search of PubMed/Medline from January 1995 until August 2016, the relevant literature on PSCI/ PSD was critically reviewed. The following keywords were employed in te search: "cognitive impairment", "dementia", "definitions and classification", "diagnosis", "neuroimaging", "interventions", "biomarkers", and "stroke". References from the selected papers published in the English language were evaluated and included if they were found to be relevant to the focus of this systematic review. Experts were divided in four groups. The first group of experts conducted research about basic concepts, definitions, and epidemiology of PSD following stroke as well as on tools for assessment of cognitive impairment. The second group evaluated biomarkers for PSD, whereas as neuroimaging studies and interventions for PSD prevention and treatment were critically reviewed by the third and fourth groups, respectively.

\section{Definitions}

A variety of classifications, diagnostic criteria, and descriptive syndromes are used to define PSCI [6], but a widely accepted and harmonized terminology is still missing [7]. Post-stroke neuropsychological syndromes overlap - PSCI is responsible for a substantial number of vascular cognitive impairment (VCI) syndromes; PSCI includes the subgroups of PSD and PSCI not fulfilling criteria for dementia.

The direct application of established diagnostic criteria for dementia may not be suited to stroke populations. For example, the differentiation of dementia from PSD and PSCI not fulfilling criteria for dementia is usually based on limitations in activities of daily living. In stroke survivors with substantial physical impairments it may be difficult to assess changes in activities of daily living related to specifically cognitive problems [8]. Usual definitions of dementia emphasize the presence of multidomain cognitive impairments, and in particular memory deficits. However, in stroke, it may be possible to have disabling cognitive problems but retain memory [9].

One of the main questions in the relationship between stroke and dementia occurring after the cerebrovascular event is whether the stroke causes the cognitive deterioration, contributes to it, or just alerted the physicians to the problem. The question remains to be answered, but undoubtedly about one-third of stroke survivors are found to have a significant degree of cognitive impairment within the first months after the event [10].

We propose the term PSD for any dementia which develops following a clinical cerebrovascular event. Although we use the descriptor "post-stroke", emerging evidence suggests that transient ischemic attack may also be associated with adverse cognitive prognosis [11]. Using the term PSD in this way does not suggest a particular underlying neuropathological process. This seems appropriate as dementia following stroke often comprises a mix of "vascular" insults and neurodegenerative processes [12]. Stroke occurs predominantly in older adults and therefore stroke patients may have pre-stroke cognitive decline of varying severity [13]. Recognizing the pre-stroke cognitive state is essential to allow appropriate classification, e.g., a patient with pre-existing cognitive impairment (diagnosed or undiagnosed) who then has a minor stroke should not be labeled as PSD.

The time assessment of cognitive impairment is another relevant diagnostic factor. Acute deficiencies in cognitive test scores are often observed following a stroke and retesting after several weeks often reveals improvements $[14,15]$. Therefore, the final diagnosis of PSD should be delayed to at least 6 months after the event. We recognize that certain strategic infarcts (for example, in dominant medial temporal lobe) are associated with immediate cognitive syndromes; however, we would still reserve the dementia diagnosis until at least 6 months. This delay in applying a dementia diagnostic label is in keeping with International classification systems such as the American Psychiatric Association Diagnostic and Statistical Manual.

The definition we have proposed is in accordance with other groups. The Vascular Impairment of 
Cognition Classification Consensus Study (VICCCS; http://www.vicccs.info/) considered PSD as a major subtype of VCI and felt that this label should be used where there is a clear temporal relationship between stroke and cognitive decline. A national Korean study of VCI epidemiology defined PSD as any major cognitive impairment seen at more than 3 months after a stroke event, regardless of pre-stroke cognitive status [16].

The terms PSD and vascular dementia $(\mathrm{VaD})$ are not synonymous. The often used term, $\mathrm{VaD}$, has evolved during the last century. Today, $\mathrm{VaD}$ represents a concept which includes not only multiple cortical and/or subcortical infarcts, but also strategic single infarcts, noninfarction white matter lesions, hemorrhages, and hypoperfusion as possible causes of dementia. $\mathrm{VaD}$ can be considered a subgroup of VCI, representing fully developed dementia after a clearly identified vascular event (the overlap of these definitions is illustrated in Fig. 1).

\section{Assessments}

International guidelines recommend cognitive and mood assessment for all stroke survivors and it is increasingly recognized that cognitive assessment should be part of the "routine" neurological examination in research and clinical practice $[17,18]$. Several cognitive assessment tools are available and there is no accepted consensus on preferred approach $[19,20]$. Stroke-specific cognitive assessment tools are available [21], but most centers still use tools developed for non-stroke populations. A systematic review of test properties in stroke did not show clear superiority with respect to global accuracy [22]. The most suitable assessment will vary with the purpose of testing; for example, if a rater wishes to ensure all potential cases of PSD are identified then a highly

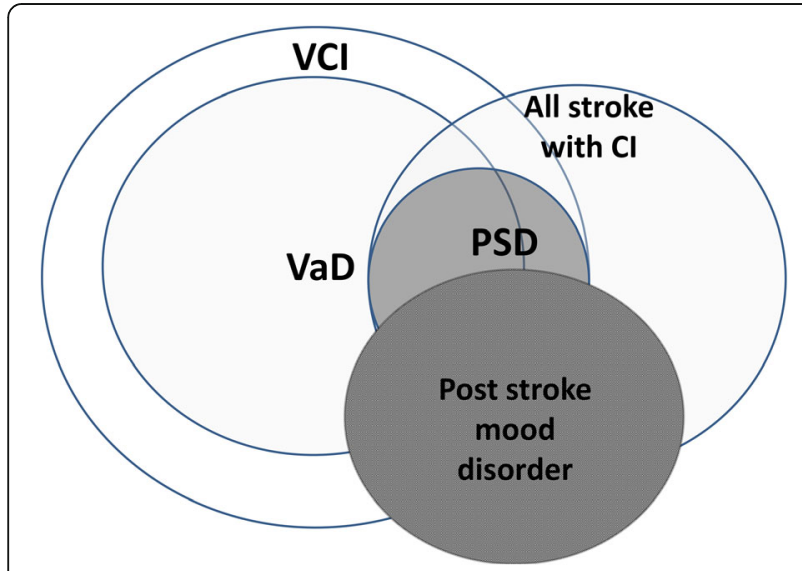

Fig. 1 Venn diagram illustrating the overlap of constructs used to define cognitive states relevant to stroke. Cl cognitive impairment, PSD post-stroke dementia, VaD vascular dementia, $\mathrm{VCl}$ vascular cognitive impairment sensitive scale such as the Montreal Cognitive Assessment (MoCA) would be preferable. The cut point used to define PSD can also be adjusted; for example, many centers recommend a lower threshold for MoCA when used in stroke settings. Choice of assessment should also be guided by other factors such as availability, familiarity, and feasibility (Table 1). Feasibility is of particular concern in acute stroke, where severity of disease may preclude lengthy neuropsychological testing [23].

Informant-based structured questionnaires can capture the patient's cognitive state before the stroke. The Informant Questionnaire for Cognitive Decline in the Elderly (IQCODE) is the most commonly employed assessment [24]; it has reasonable accuracy in determining dementia, it is available in several languages, and can be completed in minutes using the short form version [25]. IQCODE has been used for assessment of pre-stroke and post-stroke cognition and to aid prognosis; indeed, the properties of IQCODE vary according to the purpose of testing. Testing of cognition should be complemented by functional assessment. The modified Rankin Scale and Barthel Index are the most commonly used functional assessment tools [26]. Post-stroke cognitive issues will often coexist with other neuropsychological problems and should be assessed by validated tools as, for example, language disorders (Frenchay Aphasia Screening Test), mood disorder (Hospital Anxiety Depression Scale), fatigue (Fatigue Severity Scale), delirium (Confusion Assessment Method), and apathy (Apathy Evaluation Scale).

Common stroke-related impairments can complicate assessment if, for example, the patient is unable to complete a pencil and paper task if the dominant hand is weak [27]. Cognitive assessment in the presence of aphasia is particularly problematic. Tools designed for use in aphasia are available such as the Cognitive Assessment Scale for Stroke Patients, which can be administered without using language, and the Oxford Cognitive Screen [27].

\section{Epidemiology}

Stroke is recognized as one of the major causes of adult disability globally. VaD including PSD is the second most common cause of cognitive decline, with only Alzheimer disease (AD) being more prevalent [28]. The lifetime risk of developing either stroke or dementia at age of 65 is one in three in men and one in two in women [29]. With changing population demographics, increased life expectancy and improved survival from stroke, the absolute numbers of patients with PSD will increase. However, due to its relation with stroke incidence, PSD might be reduced with improved stroke prevention.

A pooled analysis of international data classified important contributors and risk factors to PSD as demographic parameters, factors related to pre-stroke functional status and to the index stroke (s), and factors 
Table 1 Properties of selected post-stoke dementia assessment scales

\begin{tabular}{lccllll}
\hline Test & Sensitivity & Specificity & Free to use & Time to administer (min) & Validated in stroke & Suitable for aphasia \\
\hline ACE-R $^{2}$ & 0.96 & 0.70 & Yes & 20 & Yes & No \\
IQCODE $^{\text {a }}$ & 0.81 & 0.83 & Yes & 5 & Partial & Yes \\
MMSE & 0.72 & 0.82 & No & 5 & Yes & No \\
MoCA $(<26 / 30)$ & 0.95 & 0.45 & Yes & 10 & Yes & No \\
MoCA $(<22 / 30)$ & 0.85 & 0.76 & Yes & 10 & Yes & No \\
R-CAMCOG & 0.81 & 0.92 & No & 15 & Yes & No
\end{tabular}

Test properties are from meta-analyses and describe accuracy at conventional thresholds unless otherwise stated

ACE- $R$ Addenbrookes Cognitive Evaluation Revised, MMSE Mini-Mental State Examination, MoCA Montreal Cognitive Assessment, R-CAMCOG Rotterdam CAMCOG

${ }^{a}$ Accuracy of IQCODE for assessment of PSD in the longer term after stroke

relating to post-stroke complications and brain imaging factors (Table 2) [30, 31].

Silent brain infarctions (SBI) are common findings in elderly patients, but their relationship to dementia remains uncertain. Bornstein et al. [32] studied admission CT scans of 175 consecutive patients without dementia, presenting with a first stroke incident. SBI were defined as CT evidence of stroke not compatible with the acute event. Stroke survivors were followed for their mental state for 5 years and the authors found that SBI did not predict the development of PSD.

According to study of Grau-Olivares and Arboix [33], ischemic cerebral small-vessel disease (SVD) should be regarded as a severe condition prodrome of subcortical $\mathrm{VaD}$, rather than a relatively benign disorder, as considered earlier, especially since SVD can be seen on MRI studies (white matter lesions, lacunes, and microbleeds). Recent studies showed that the proportion of dementia caused by SVD ranges from 36 to $67 \%[9,28,31,33]$. Further, patients with a first-ever lacunar stroke present

Table 2 Risk factors for PSD [30, 31]

\begin{tabular}{ll}
\hline Demographic factors & Age (over 65 years) \\
& Lower educational level \\
& Female sex \\
& Non-Caucasian origin \\
& Physical impairment \\
Pre-stroke factors & Cognitive decline \\
Index stroke factors & Hemorrhagic stroke \\
& Supratentorial stroke location \\
& Dominant hemisphere stroke \\
Post-stroke factors & Recurrent strokes \\
Infection & Delirium \\
Neuroimaging factors & Early seizures \\
& Cerebral small-vessel disease \\
& Cortical atrophy \\
& Medial temporal lobe atrophy
\end{tabular}

with cognitive impairment in more than half of cases and more than $55 \%$ of patients fulfill mild cognitive impairment criteria [33]. Additionally, the presence of silent multiple lacunar infarctions in patients with firstever lacunar stroke is an independent predictor of poor performance on executive functions and short delayed verbal memory tests. Thus, in the initial stages of SVD, mild neuropsychological abnormalities appear to be related to lacunes rather than to perivascular hyperintensities of vascular cause or leukoaraiosis [34].

A number of important prospective cohort studies have documented cognitive change following stroke [35, 36]. Meta-analysis of these studies suggests that some form of cognitive impairment affects up to one third of stroke survivors; this figure does not differentiate stroke etiology and may include a proportion (estimated at around 10\%) with pre-stroke dementia. Prevalence of PSCI is higher in those with recurrent stroke events [31]. These data might underestimate the true prevalence of post-stroke cognitive change as patients included in studies tend to exclude those with larger strokes who are unable to consent or attend follow-up visits [37]. There are also issues with feasibility of cognitive testing and attrition to follow-up that will contribute to under-estimation of the true burden of cognitive impairment [38].

PSD is often recognized in the first weeks to months post ictus and, thereafter, prevalence of PSD increases with time. Compared to controls with no stroke, incident stroke is associated with acute change in cognition and change in rate of temporal cognitive decline [39]. Pooled analyses of longitudinal cohorts suggest a linear increase of 3 and $1.7 \%$ per year in hospital- and population-based studies, respectively [31]. In contrast to cognitive changes in the normal aging population, a linear rate of change is not observed, but stroke survivors seem to show different cognitive "trajectories" and these trajectories may change over time (Fig. 2).

\section{Biomarkers and predictors of PSD}

Biomarkers for PSD may include neuroimaging measures and metabolic, genetic, and inflammatory mediators [40]. 


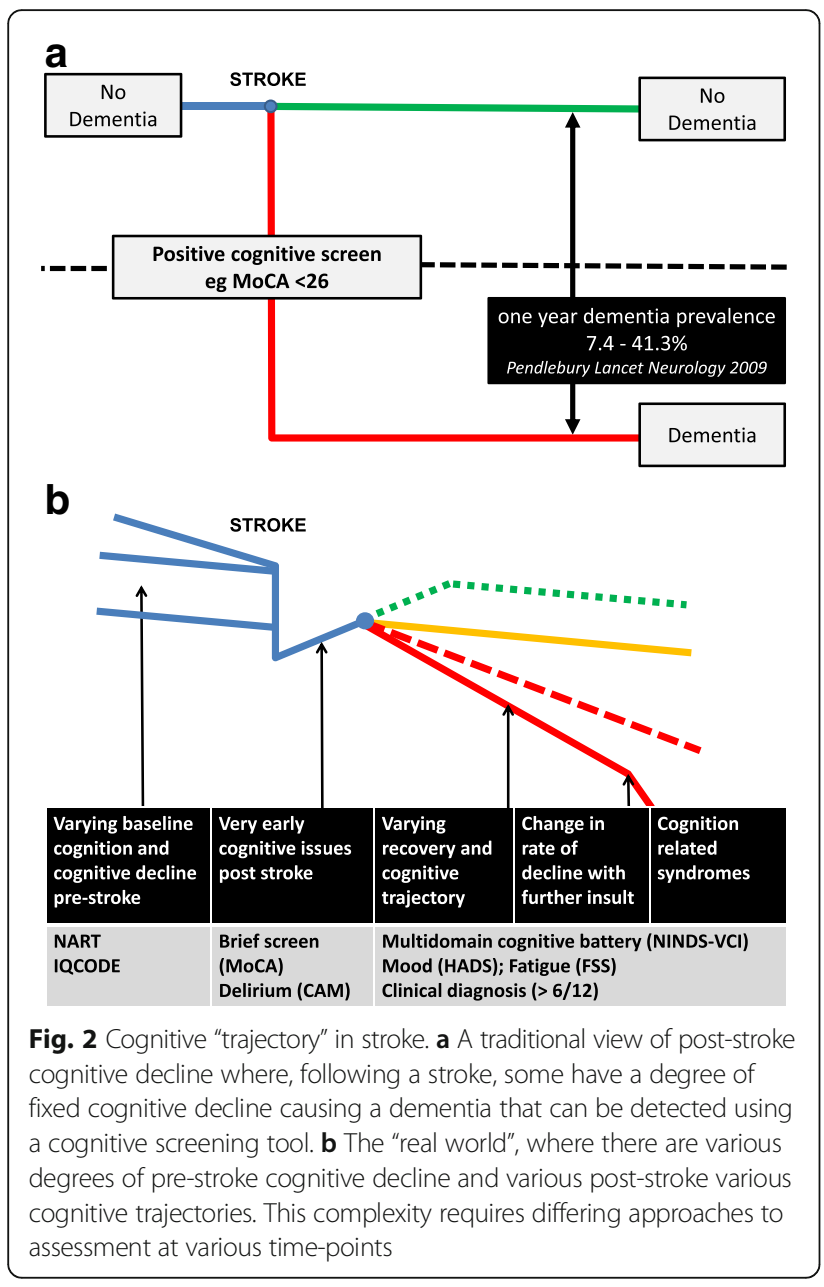

Both neurodegenerative and vascular mechanisms are activated and probably result in overlapping processes, therefore sharing some pathological features and likely having some common biomarkers.

\section{Genetic markers}

Genes underlying PSD and VCI may include those shared by $\mathrm{VaD}$ and $\mathrm{AD}$, namely genes involved in the pathways of amyloid production or elimination (apolipoprotein E and possibly presenilin and APP) may confer susceptibility to develop dementia after vascular brain injury, thus explaining, at least in part, the well-known interaction and synergism between $\mathrm{VaD}$ and $\mathrm{AD}$. Polymorphisms (SNPs) in genes reported as conferring susceptibility to develop PSD and VaD include the e4 allele of apolipoprotein E, a well-known risk factor for $\mathrm{AD}$, although its association with $\mathrm{VaD}$ and PSD has been controversial [41-47]. Another reported SNP is in the gene encoding the angiotensin-converting enzyme, one of the enzymes in the renin-angiotensin system. Studies investigating the angiotensin-converting enzyme SNP as a predictor for post-stroke cognitive decline have yielded controversial data [48-52]. A genome-wide association study in the Rotterdam cohort revealed a genetic locus associated with $\mathrm{VaD}$, located on the $\mathrm{X}$ chromosome close to the androgen receptor gene [53]. An additional SNP is endothelial nitric oxide synthase, reported as associated with incident dementia in elderly stroke survivors [54]. The main research areas currently being investigated are genetic predisposition, predominantly in genes linked to both AD and vascular disease and inflammatory response.

\section{Cerebrospinal fluid (CSF) biomarkers}

So far, the search for a precise and reliable biochemical indicator which would help establish the diagnosis of $\mathrm{VaD}$ and PSD has not been productive. Although, the A $\beta-42$ peptide and tau protein CSF levels are sensitive markers of $\mathrm{AD}$, they showed the lowest specificity in $\mathrm{VaD}$ [55]. Promising results were obtained measuring the levels of matrixdegrading metalloproteinases (MMPs) in CSF, such as gelatinase A (MMP-2) and gelatinase B (MMP-9) [56, 57]. Gelatinases interfere with molecules of the basal lamina, leading to proteolytic disruption of the blood brain barrier (BBB). Further, they are involved in myelin breakdown. Brain ischemia induces a gelatinase $\mathrm{A}$ and $\mathrm{B}$ elevation at different time points after the stroke. Recent studies showed that the MMP-9 CSF level is significantly higher in $\mathrm{VaD}$ patients compared to $\mathrm{AD}$ patients as well as elderly healthy subjects, and may help in distinguishing $\mathrm{AD}$ from $\mathrm{VaD}$ [56]. Other CSF markers also reported as associated with $\mathrm{VaD}$, and indirectly PSD, are levels of $\alpha-1$ antitrypsin, plasminogen activator inhibitor-1, and apolipoprotein $\mathrm{H}[58]$.

\section{Serum biomarkers}

Serum biomarkers, such as $\beta$ secretase enzyme and receptor levels for advanced glycation end products, have been suggested to correlate with early PSCI [45]. Homocysteine, vitamin B12, and folic acid levels were linked to PSCI; however, supplementation with B vitamins had no effect on the incidence of cognitive impairment or decline [59].

\section{Inflammatory mediators}

Inflammation in stroke seems to have both detrimental and beneficial effects. Activation of resident cells (microglia, astrocytes, and endothelial cells) appears to be neuroprotective and promotes brain regeneration/recovery, whereas recruitment of immune cells with a subsequent increase in the expression of inflammatory mediators, such as reactive oxygen species, cytokines, and chemokines, together with $\mathrm{BBB}$ disruption, could lead to edema and neuronal death [60]. Chronic activation of innate immune responses can trigger neurotoxic pathways leading to progressive degeneration. Damaged neurons 
themselves may also exacerbate immune-mediated disease by release of chemokines and activation of microglia [61]. Several recent longitudinal studies have investigated the relationship between inflammatory markers and PSD, but an association is not yet established. Erythrocyte sedimentation rate (ESR), C-reactive protein, interleukin 6 , and interleukin 12 were suggested as predictors of PSCI [62, 63]. Recently, Kliper et al. [64] showed strong relationship between ESR and cognitive performance among stroke survivors, where higher ESR levels were associated with worse performance in cognitive tests, particularly memory scores. Findings from the TABASCO study have raised the possibility that many individuals with cerebrovascular ischemic events harbor a long and persistent pro-inflammatory background [64]. Whether lowering inflammation can also prevent PSD needs to be addressed in prospective clinical trials.

\section{Peripheral microRNA (miRNA) profiles as novel biomarkers}

miRNAs are a type of small non-coding RNA molecules that act as endogenous regulators of gene expression by binding to complementary sequences of target messenger RNA. The dysregulation of miRNAs is thought to be involved in many diseases, and changes in the miRNA expression profile have been observed in cerebral ischemia and AD. Studies have demonstrated that serum miRNAs miR-93, miR-146a, and miR-31 were significantly up regulated in $\mathrm{VaD}$ compared to the controls; thus, they could serve as specific biomarkers to discriminate $\mathrm{AD}$ from $\mathrm{VaD}$ [65]. Endogenous circulating miRNAs have been found to be stable and consistently detected in serum, plasma and other body fluids because of their packaging and secretion into the blood within exosomes. Currently, information regarding miRNAs in $\mathrm{VaD}$ and PSD studies is limited, but the potential diagnostic value of miRNAs is very promising. In conclusion, no molecular biomarkers have been confirmed as being specifically associated with PSD.

\section{Neuroimaging}

Neuroimaging in PSD provides significant information about the anatomical substrate of the disorder and has an important role in PSD diagnosis. Further, it adds to prediction of cognitive decline after stroke; for example, hippocampal atrophy is a strong predictor for PSD outcome $[12,66]$. Most acute stroke patients undergo CT brain imaging and, therefore, CT studies are representative of the whole clinical population. In everyday practice, CT is performed mainly to exclude intracerebral hemorrhage and certain stroke mimics (e.g., brain tumors), and can often show early signs of ischemia, as well as old stroke lesions. Additionally, the presence and extension of cerebral atrophy and white matter lesions can easily be seen on brain CT scans. Such neuroimaging characteristics may help predict subsequent cognitive impairment $[67,68]$.

MRI represents the most significant neuroimaging modality in PSD. If not contraindicated, MRI, rather than CT, is preferred for daily, routine clinical use as well as in research studies since it has higher specificity and sensitivity for detecting pathological substrates [66]. Neuroimaging standards with a generally accepted terminology allowing comparison of findings between different centers have been suggested (STandards for ReportIng Vascular changes on nEuroimaging, STRIVE) [69]. A large number of studies recognized MRI signs of cerebral SVD (lacunes, white matter hyperintensities, microbleeds, silent infarcts, white matter changes), as well as global cerebral atrophy and medial-temporal lobe atrophy as determinants and predictors of PSD. Vascular lesions associated with PSD are mainly found in subcortical brain areas, especially including sub-frontal and orbitofrontal white matter circuits. These lesions include single infarction in strategic areas such as the dominant thalamus or angular gyrus, deep areas of frontal lobe, and the left hemisphere, as well as brain infarcts in both hemispheres and volume-driven infarctions reaching a critical threshold of brain tissue loss or injury [70]. Additionally to size and location of the vascular lesion, the involvement of functional network fiber tracts, assessed by diffusion tensor imaging, may be crucial for cognitive impairment after stroke; its role is still being studied [71]. Although brain atrophy is a frequent finding in cerebral SVD, the pathophysiological mechanisms are not fully elucidated. In a recent study, Duering et al. [72] showed that cortical neurodegeneration following ischemia in the subcortical region probably represents pathophysiological mechanisms for cerebral atrophy in cerebrovascular disease; they showed that subcortical infarcts trigger focal thinning in connected cortical areas. Additionally, patients with mild VCI showed clear progressive gray matter atrophy in cortical (temporal and frontal) and subcortical (pons, caudate and cerebellum) regions after first-ever lacunar stroke, in contrast to patients without initial cognitive impairment [73].

Mild to moderate stroke patients with pre-existing white matter lesions are more vulnerable to cognitive impairment regardless of their new ischemic lesions [74]. Results from the SMART-MR study suggested that the interaction between brain atrophy and white matter hyperintensities or infarcts could aggravate cognitive decline [75]. There is evidence of the role of hippocampal mean diffusivity in the post-stroke cognitive state, above and beyond that of volume and connectivity of this structure [76].

Imaging of cerebral blood flow and cerebral glucose metabolism, by FDG-PET in particular, has been applied 
for several years in the differentiation between dementias [77]. However, functional and molecular imaging has a special role for research on the pathophysiology and the factors involved in development of PSD. Stroke and the deposition of amyloid in the cerebral cortex are both known risks for developing dementia [78, 79]. While animal models suggest that stroke-induced inflammation and amyloid deposition act synergetically [79], this relationship remains to be established in human stroke. Experimentally, large vessel infarcts or small striatal infarcts are larger in the presence of extracellular amyloid beta $\left(\mathrm{A}_{\beta}\right)$ deposits, which are fundamental markers of AD. Patients with minor cerebral strokes and moderate $\mathrm{AD}$ lesions will develop the clinical manifestations of dementia. A reasonable question is whether the stroke was just a signal and not necessarily a player in the pathogenesis of cognitive deterioration. Additionaly, small striatal infarcts in the presence of high levels of amyloid in the brain exhibit a progression in infarct size over time with an enhanced degree of cognitive impairment, AD-type pathology, and neuroinflammation compared with striatal infarcts or high amyloid levels alone. Recently, it has been stated that stroke is a specific vascular risk factor for cognitive impairment [80]. There is an overlap between VCI risk factors (hypertension, diabetes, and atherosclerosis) and those for stroke, as well as $\mathrm{AD}$ risk factors. Stroke may alter the clinical expression of a given load of AD pathology, but more studies are needed for definitive clarification of the relationships between stroke, $\mathrm{AD}$, and cognitive detoriation.

\section{Interventions}

The best way to prevent PSD is to prevent stroke recurrence and stroke severity through optimal acute treatment and intensive secondary prevention. Intravenous and intra-arterial interventions (if indicated), treatment in the stroke unit including prevention of complications, and early rehabilitation are thought to limit the damage from the stroke lesion and improve outcome [81]. Secondary prevention includes medical interventions and lifestyle modification. To date, there is limited evidence for specific therapeutic strategies for preventing cognitive decline after stroke. However, aggressive treatment of vascular risk factors may potentially prevent PSD through prevention of the total vascular burden, including progression of vascular disease and vascular risk associated with dementia. An observational study showed reduced cognitive impairment in patients with appropriate vascular risk management post-stroke [82]; this included antiplatelet therapy, antihypertensive drugs and statins, or anticoagulants, if indicated. In addition, cognitive function can benefit from treatment of neuropsychiatric symptoms like depression, apathy, and anxiety, as well as cognitive training/stimulation [83].
In a pilot study, the relationship between amyloid deposition, microglia activation, and cognitive performance in stroke patients was investigated [84]. Preliminary results suggest that cortical amyloid deposition and post-stroke white matter inflammation contribute to PSD, indicating that various pathophysiological mechanisms are involved. If confirmed in larger trials, this finding might offer possibilities for clinical intervention to prevent post-stroke cognitive decline by modulation of inflammation or amyloid deposition. In addition, endothelin dysfunction with secondary neuroinflammation seen in acute non-atherothrombotic etiology stroke can be a potential target for drug treatment [85].

\section{Pharmacological interventions for prevention of PSD} Blood pressure lowering

A review by Zanchetti et al. [86] of randomized controlled trials (RCTs) not limited to stroke patients suggests that blood pressure lowering may have some beneficial effects on cognitive decline. Hypertension treatment after stroke preserves cognition through prevention of recurrent stroke, but it is not yet clear whether it prevents cognitive decline through other mechanisms. Thus far, only four published RCTs restricted to patients with a previous stroke tested the effect of antihypertensive drugs on cognition as secondary outcome. The Perindopril Protection Against Recurrent Stroke study showed that long-term blood pressure lowering after stroke with perindopril was associated with reduced cognitive decline [87]. This effect was more pronounced in patients with recurrent stroke, suggesting a beneficial effect due to secondary stroke prevention. In contrast, telmisartan did not change cognition in the Prevention Regimen for Effectively Avoiding Second Strokes (PRoFESS) study [88]. Further, results of the Secondary Prevention of Small Subcortical Strokes (SPS3) trial showed no differences in cognitive function amongst groups according to blood pressure control or antiplatelet drug used [89]. One study, with cognition as the primary outcome, did not find any association between cognitive test performance and different blood pressure goals [90]. Similarly, candesartan did not improve cognitive function at 6 months when compared to placebo in the Scandinavian Candesartan Acute Stroke Trial (SCAST) [91].

\section{Statin treatment}

Statin therapy in secondary stroke prevention has shown an effect on new vascular events but only marginally reduces the risk of stroke recurrence [92]. Statins lower LDL cholesterol and may have a beneficial effect on platelet function, endothelial activity, and inflammation [93]. No study has tested the effect of statins on cognition in patients with previous stroke. However, two large studies (HPS and PROSPER), which included patients 
with risk factors for, or a history of, vascular diseases, failed to show that either simvastatin or pravastatin affected cognition [94, 95].

\section{Other potential pharmacological interventions}

Many therapeutic strategies have been developed for neuroprotection in acute stoke. Nitric oxide donors are thought to reduce infarct size and improve cerebral blood flow, but did not improve cognitive function poststroke [96]. Acetylcholinesterase inhibitors and memantine may have a beneficial effect on cognition in patients with mild to moderate vascular dementia in general [97], but evidence for both general improvement are limited. Cerebrolysin had a beneficial effect on global outcome, but did not assess cognition, especially in stroke patients [98]. Selective serotonin reuptake inhibitors may be associated with overall recovery after stroke even in the absence of depression [99]. Fluoxetine is proposed to improve stroke recovery, and there is an ongoing trial that includes cognition as an outcome [100]. The dietary nucleotide citicoline may also improve recovery [101]. There are ongoing studies to explore the potential positive effect of phosphodiesterase-3, anti-inflammatory agents, BBB modulators, endothelin antagonist, flavonoids, immunosuppressive agents, peroxisome proliferator-activated receptor gamma antagonists, prostacylin, sympathomimetics, xanthine oxidase inhibitors, antidepressants, neurotrophic agents, gingko biloba extract Egb-761, and others [12, 102].

\section{Lifestyle interventions}

\section{Multifactorial interventions}

Most vascular risk factors are modifiable. Modifications of lifestyle factors include physical exercise, healthy diet, moderate alcohol consumption, and smoking cessation. Multimodal lifestyle interventions have been successful in changing the lifestyle habits of stroke survivors [103, 104]. Two randomized trials have been performed with multifactorial interventions including optimal medical treatment for secondary prevention in addition to physical activity, diet, weight control, smoking cessation, healthy diet with better glycemic control, and cognitive stimulation. One tested the effect of the multimodal risk factor intervention over 1 year on cognition, but no change in cognitive outcome was found [105]. However, significant beneficial effects were found on depression as the secondary outcome [106]. The recently published RCT by Matz et al. [107] showed that stroke patients receiving multiple, intensive life-style interventions did not differ in cognitive outcomes after 2 years compared to patients who received standard care; a small non-significant reduction in dysexecutive syndromes appearing in the intervention group was noted, but not significant. This finding indicates that studies for lifestyle interventions have to be performed in larger populations with neuropsychological functions as outcome measures, particularly the executive domains.

\section{Physical interventions}

A systematic review including nine studies, published in 2012, showed that increased physical activity after stroke improves cognitive performance [108]. Exercise training is standard for cardiovascular disease management, and cardiac rehabilitation was shown to improve cognitive performance in several studies [109]. Sharing the same risk factors as stroke, brain rehabilitation including exercise might be a right strategy in future stroke care, supported by papers published over the past 2 years. A 6-month training model combining aerobic and resistance training, initiated at least 10 weeks post-stroke, resulted in improved cognitive function and reduction of patients meeting the threshold criteria for mild cognitive impairment [110]. A recently published paper by Moore et al. [111] showed that exercise therapy three times per week for 19 weeks improved cognitive function, increased regional blood flow in the medial temporal lobe, and prevented structural loss in the same region. An ongoing RCT, Move IT, aims to investigate whether a physical exercise program can prevent cognitive decline in the acute phase after transient ischemic attack or minor ischemic stroke [112].

\section{Cognitive training}

Cognitive rehabilitation is a complex therapeutic intervention aiming to improve cognitive function after stroke; at present, the approached strategies are individual remediation therapy with a neuropsychologist, group-based training, and computer cognitive training [113]. Cognitive rehabilitation plays an integral role in stroke multidisciplinary rehabilitation and should be started shortly after stroke onset, with effectiveness both in the post-acute period and a few years after stroke [114]. However, there are insufficient data to conclude on the long lasting benefits in memory and executive function for post-stroke survivors [114, 115]. Moreover, data is missing regarding whether the possible improvements in neuropsychological tests are related to improvement in everyday functional activities [116].

\section{Non-invasive brain stimulation}

Non-invasive brain stimulation techniques, including repetitive transcranial magnetic stimulation and transcranial direct current stimulation, have been reported to improve functional status of stroke patients through modulation of the excitability of cortical circuits $[117,118]$. Nevertheless, most previous studies included a small number of participants and evaluated only the short-term effect. Therefore, there is limited evidence for recommendation of repetitive transcranial magnetic stimulation or transcranial direct 
current stimulation in the treatment of PSCI. Future studies are needed to elucidate appropriate stimulation sites, optimal parameters, and stimulation protocols to overcome individual differences such as skull shape or genetic polymorphisms [119].

\section{Actovegin}

Natural biological products, such as actovegin, may have beneficial effects in the restorative phase of ischemia [120]. Elmilnger et al. [121] found protective and antioxidative effects of the hemodialysate actovegin on primary rat neurons in vitro. The efficacy of actovegin was investigated by Mikhailova et al. [122] in the treatment of mild cognitive impairment post-stroke. The authors found a positive therapeutic effect, including increased speed of mental processes and reduced memory impairment as well as a positive impact on asthenic and depressive symptoms. In addition, the ARTEMIDA study explored whether this treatment has a disease-modifying effect on PSD [123]. Actovegin has been shown to have effects on some cellular processes in the aging brain and recent experimental studies revealed actovegin to play a role in neuroprotective mechanisms [124].

\section{Conclusions and further prospects}

A variety of classifications, diagnostic criteria, and descriptive syndromes are used to define post-stroke cognitive problems, but a widely accepted and harmonized terminology is still missing. We propose the term PSD for any dementia following stroke that has a temporal relationship with stroke.

The best way to prevent PSD is optimal treatment to reduce stroke incidence and severity and, once stroke occurs, intensive prevention of early complications and long-term stroke recurrence. To date, there is limited evidence for specific therapeutic strategies for preventing cognitive decline after stroke. However, aggressive treatment of vascular risk factors, such as antiplatelet therapy, antihypertensives and statins, or anticoagulant therapy (if indicated), may potentially prevent PSD through inhibition of the total vascular burden, including progression of vascular disease and vascular risk associated with dementia. In addition, cognitive function can benefit from treatment of neuropsychiatric symptoms like depression, apathy, and anxiety, as well as cognitive training/stimulation.

In the future, it is necessary to introduce a uniform definition of cognitive impairment state following stroke, which should be widely accepted, leading to more accurate epidemiological results and a greater potential to compare and pool data. For this to be possible, all inclusion and exclusion criteria should be precisely defined, and in particular the time assessment of cognitive impairment following stroke. We propose that final diagnosis of PSD should be delayed to at least 6 months after the event.

One of the main strategies for easier identification of PSD is finding a precise biochemical marker. Since there is still no reliable marker, the search continues, with an enormous influence on future health economics and the development of preventative treatments and strategies. Therefore, multi-center studies on long-term cognitive outcomes in stroke patients should be given high priority.

\section{Availability of data and materials}

Data sharing is not applicable to this article as no datasets were generated or analyzed during the current study.

\section{Authors' contributions}

All authors contributed to the drafting and writing the manuscript,

discussions and critical amendments, analysis and interpretation, literature search, data extractions, and critical revision of the manuscript for important intellectual content. All authors read and approved the final manuscript.

\section{Competing interests}

The authors declare that they have no competing interests.

\section{Author details}

${ }^{1}$ Neurology Clinic, Clinical Center of Serbia, School of Medicine, University of Belgrade, Dr Subotica 6, 11000 Belgrade, Serbia. ${ }^{2}$ Department of Clinical Neurosciences and Preventive Medicine, Danube University Krems, Krems, Austria. ${ }^{3}$ Max Planck Institute for Metabolism Research, Cologne, Germany. ${ }^{4}$ Institute of Cardiovascular and Medical Sciences, University of Glasgow, Glasgow, UK. ${ }^{5}$ Department of internal medicine, Oslo University Hospital, Ullevål and Department of Medical Research, Bærum Hospital, Vestre Viken Hospital Trust, Bærum, Norway. ${ }^{6}$ Department of Neurology, University Hospital Essen, Essen, Germany. ${ }^{7}$ Stroke Unit, Department of Neurology, Tel-Aviv Sorasky Medical Center, Tel-Aviv, Israel. ${ }^{8}$ Shaare Zedek Medical Center, Jerusalem, Israel. ' Department of Neurology, Radboud University Medical Center, Donders Institute for Brain, Cognition and Behaviour, Nijmegen, The Netherlands. ${ }^{10}$ Department of Neurology and Neurosurgery, McGill University at SMBD Jewish General Hospital and Lady Davis Institute for Medical Research, Montreal, Québec, Canada. ${ }^{11}$ Department of Rehabilitation Medicine, Pusan National University School of Medicine, Busan, Republic of Korea. ${ }^{12}$ Department of Physical and Rehabilitation Medicine, Sungkyunkwan University School of Medicine, Center for Prevention and Rehabilitation, Heart Vascular and Stroke Institute, Samsung Medical Center, Seoul, Republic of Korea. ${ }^{13}$ Department of Neurology, Inha University School of Medicine, Incheon, South Korea. ${ }^{14}$ Hallym University Medical Center, Kang Nam Sacred Heart Hospital, Seoul, South Korea. ${ }^{15}$ Department of Neurology, Gachon University Gil Medical Center, Incheon, South Korea. ${ }^{16}$ Department of Neurology, University Clinical Center Tuzla, School of Medicine University of Tuzla, 75000 Tuzla, Bosnia and Herzegovina. ${ }^{17}$ Department of Internal Medicine, University of Michigan and the VA Ann Arbor Healthcare System, Ann Arbor, MI, USA. ${ }^{18}$ Department of Neurology, Rambam Health Care Campus, Haifa, Israel. ${ }^{19}$ Technion Faculty of Medicine, Haifa, Israel. ${ }^{20} \mathrm{Centre}$ for Clinical Brain Sciences, University of Edinburgh, Edinburgh, UK. ${ }^{21} \mathrm{Clinic}$ of Neurology, Clinical Center Nis, Nis, Serbia. ${ }^{22}$ U1171-Department of Neurology, University of Lille, Inserm, Faculty of Medicine, Lille University Hospital, Lille, France. ${ }^{23}$ Department of Neurology and Medical Rehabilitation, Krasnoyarsk State Medical University named after Professor V.F. Voyno-Yasenetsky, Krasnoyarsk, Russia. ${ }^{24}$ Department of Neurosurgery, Clinical Hospital CenterZemun, Belgrade, Serbia. ${ }^{25}$ Department of Clinical Neurosciences, "Iuliu Hatieganu" University of Medicine, Clij-Napoca, Romania. ${ }^{26}$ Department of Neurology, Tel Aviv University, Ramat Aviv 69978, Israel.

Received: 13 September 2016 Accepted: 3 January 2017

Published online: 18 January 2017

\section{References}

1. Strong K, Mathers C, Bonita R. Preventing stroke: saving lives around the world. Lancet Neurol. 2007;6:182-7. 
2. Lees R, Fearon P, Harrison JK, Broomfield NM, Quinn TJ. Cognitive and mood assessment in stroke research: focused review of contemporary studies. Stroke. 2012;43:1678-80.

3. McKevitt C, Fudge N, Redfern J, et al. Self-reported long term needs after stroke. Stroke. 2011;42:1398-3.

4. Pollock A, St George B, Fenton M, Firkins L. Top ten research priorities relating to life after stroke. Lancet Neurol. 2012;11:209.

5. Fride $Y$, Adamit $T$, Maeir A, Ben Assayag E, Bornstein NM, Korczyn AD, Katz N. What are the correlates of cognition and participation to return to work after first ever mild stroke? Top Stroke Rehabil. 2015;22(5):317-25.

6. Ritchie CW, Muniz Terrera G, Quinn TJ. Dementia trials and dementia tribulations: methodological and analytical challenges in dementia research. Alzheimers Res Ther. 2015;7:31.

7. Pendlebury ST, Mariz J, Bull L, Mehta Z, Rothwell PM. Impact of different operational definitions on mild cognitive impairment rate and MMSE and MoCA performance in transient ischaemic attack and stroke. Cerebrovasc Dis. 2013;36:355-62.

8. Ihle-Hansen $\mathrm{H}$, Thommessen B, Wyller TB, Engedal K, Øksengård AR, Stenset V, Løken K, Aaberg M, Fure B. Incidence and subtypes of $\mathrm{MCl}$ and dementia 1 year after first-ever stroke in patients without pre-existing cognitive impairment. Dement Geriatr Cogn Disord. 2011;32:401-7.

9. Erkinjuntti T, Gauthier S. Diagnosing vascular cognitive impairment and dementia: concepts and controversies. In: Wahlund L, Erkinjuntti T, Gauthier S, editors. Vascular cognitive impairment in clinical practice. Cambridge: Cambridge University Press; 2009. p. 3-10.

10. Gorelick PB, Nyenhuis D. Stroke and cognitive decline. JAMA. 2015;314(1):29-30. doi:10.1001/jama.2015.7149.

11. Van Rooij FG, Kessels RP, Richard E, De Leeuw FE, van Dijk EJ. Cognitive impairment in transient ischemic attack patients: a systematic review. Cerebrovasc Dis. 2016;42(1-2):1-9.

12. Brainin M, Tuomileheto J, Heiss WD, et al. Post-stroke cognitive decline: an update and perspectives for clinical research. Eur J Neurol. 2015;22:229-38

13. Henon $\mathrm{H}$, Pasquier $\mathrm{F}$, Durieu l, et al. Pre-existing dementia in stroke patients: baseline frequency, associated factors, and outcome. Stroke. 1997:28:2429-36.

14. Wagle J, Farner L, Flekkøy K, et al. Early post-stroke cognition in stroke rehabilitation patients predicts functional outcome at 13 months. Dement Geriatr Cogn Disord. 2011;31:379-7.

15. Lees R, Stott DJ, Quinn TJ, Broomfield NM. Feasibility and diagnostic accuracy of early mood screening to diagnose persisting clinical depression/anxiety disorder after stroke. Cerebrovasc Dis. 2014;37:323-9.

16. Yu KH, Cho SJ, Oh MS, Jung S, Lee JH, Shin JH, Koh IS, Cha JK, Park JM, Bae HJ, Kang Y, Lee BC, Korean-Vascular Cognitive Impairment Harmonization Standards Study Group. Cognitive impairment evaluated with vascular cognitive impairment harmonization standards in a multicenter prospective stroke cohort in Korea. Stroke. 2013;44:786-8.

17. Hachinski V, ladecola C, Petersen RC, Breteler MM, Nyenhuis DL, Black SE, et al. National Institute of neurological disorders and stroke Canadian stroke network vascular cognitive impairment harmonization standards. Stroke. 2006;37:2220-41

18. Royal College of Physicians. National clinical guidelines for stroke clinical effectiveness and evaluation unit. London: Royal College of Physicians; 2012.

19. Lees R, Corbet S, Johnston C, Moffitt E, Shaw G, Quinn TJ. Test accuracy of short screening tests for diagnosis of delirium or cognitive impairment in an acute stroke unit setting. Stroke. 2013;44:3078-3.

20. Ferris S. Report of the working group on clinical global measures. Position paper from the International working group on harmonization of dementia drug guidelines. Alzheimer Dis Assoc Disord. 1997;11 Suppl 3:8-18.

21. Demeyere N, Riddoch MJ, Slavkova ED, Bickerton WL, Humphreys GW. The Oxford Cognitive Screen (OCS): validation of a stroke-specific short cognitive screening tool. Psychol Assess. 2015;27:883-94.

22. Lees R, Selvarajah J, Fenton C, Pendlebury ST, Langhorne P, Stott DJ, Quinn TJ. Test accuracy of cognitive screening tests for diagnosis of dementia and multidomain cognitive impairment in stroke. Stroke. 2014;45:3008-18.

23. Pendlebury ST, Klaus SP, Thomson RJ, Mehta Z, Wharton RM, Rothwell PM, Oxford Vascular Study. Methodological factors in determining risk of dementia after transient ischemic attack and stroke: (III) applicability of cognitive tests. Stroke. 2015;46:3067-73.

24. Tang WK, Chan SSM, Chiu HFK, Wong KS, Kwok TCY, Mok V, et al. Can IQCODE detect poststroke dementia? Int J Geriatr Psychiatry. 2003;18:706-10.
25. Harrison JK, Fearon P, Noel-Storr AH, McShane R, Stott DJ, Quinn TJ. IQCODE for diagnosis dementia in secondary care settings. Cochrane Database Syst Rev. 2015;3:CD010772

26. Quinn TJ, Dawson J, Walters MR, Lees KR. Functional outcome measures in contemporary stroke trials. Int J Stroke. 2009;3:200-5.

27. Lees RA, Hendry Ba K, Broomfield N, Stott D, Larner AJ, Quinn TJ. Cognitive assessment in stroke: feasibility and test properties using differing approaches to scoring of incomplete items. Int J Geriatr Psychiatry. 2016. Ahead of print. doi:10.1002/gps.4568.

28. Seshadri S, Wolf PA. Lifetime risk of stroke and dementia: current concepts, and estimates from the Framingham study. Lancet. 2007;3:1106-14.

29. Henon H, Pasquier F, Leys D. Poststroke dementia. Cerebrovasc Dis. 2006;22:61-70.

30. Pasquier F, Hénon H, Leys D. Risk factors and mechanisms of post-stroke dementia. Rev Neurol. 1999;155(9):749-53.

31. Pendlebury ST, Rothwell PM. Prevalence, incidence, and factors associated with pre-stroke and post-stroke dementia: a systematic review and meta-analysis. Lancet Neurol. 2009;8:1006-18.

32. Bornstein NM, Gur AY, Treves TA, Reider-Groswasser I, Aronovich BD, Klimovitzky SS, Varssano D, Korczyn AD. Do silent brain infarctions predict the development of dementia after first ischemic stroke? Stroke. 1996;27(5):904-5.

33. Grau-Olivares M, Arboix A. Mild cognitive impairment in stroke patients with ischemic cerebral small-vessel disease: a forerunner of vascular dementia? Expert Rev Neurother. 2009;9(8):1201-17.

34. Blanco-Rojas L, Arboix A, Canovas D, Grau-Olivares M, Oliva Morera JC, Parra O. Cognitive profile in patients with a first-ever lacunar infarct with and without silent lacunes: a comparative study. BMC Neurol. 2013;13:203.

35. Ben Assayag E, Korczyn AD, Giladi N, Goldbourt U, Berliner AS, Shenhar-Tsarfaty S, Kliper E, Hallevi H, Shopin L, Hendler T, Baashat DB, Aizenstein O, Soreq H, Katz N, Solomon Z, Mike A, Usher S, Hausdorff JM, Auriel E, Shapira I, Bornstein NM. Predictors for poststroke outcomes: the Tel Aviv brain acute stroke cohort (TABASCO) study protocol. Int J Stroke. 2012;7:341-7.

36. Wollenweber FA, Zietemann V, Rominger A, Opherk C, Bayer-Karpinska A, Gschwendtner A, Coloma Andrews L, Bürger K, Duering M, Dichgans M. The determinants of dementia after stroke (DEDEMAS) study: protocol and pilot data. Int J Stroke. 2014;9:387-92.

37. Pendlebury ST, Chen PJ, Welch SJ, Cuthbertson FC, Wharton RM, Mehta Z, Rothwell PM, Oxford Vascular Study. Methodological factors in determining risk of dementia after transient ischemic attack and stroke: (I) impact of baseline selection bias. Stroke. 2015;46:641-6.

38. Pendlebury ST, Chen PJ, Welch SJ, Cuthbertson FC, Wharton RM, Mehta Z, Rothwell PM, Oxford Vascular Study. Methodological factors in determining risk of dementia after transient ischemic attack and stroke: (II) effect of attrition on follow-up. Stroke. 2015:46:1494-500.

39. Levine DA, Galecki AT, Langa KM, Unverzagt FW, Kabeto MU, Giordani B, Wadley VG. Trajectory of cognitive decline after incident stroke. JAMA. 2015;314(1):41-51

40. Bornstein NM, Aronovich B, Korczyn AD, Shavit S, Michaelson DM Chapman J. Antibodies to brain antigens following stroke. Neurology. 2001;56(4):529-30.

41. Hsiung GY, Sadovnick AD, Feldman H. Apolipoprotein E epsilon4 genotype as a risk factor for cognitive decline and dementia: data from the Canadian study of health and aging. CMAJ. 2004;171:863-7.

42. Jin YP, Ostbye T, Feightner JW, Di Legge S, Hachinski V. Joint effect of stroke and APOE4 on dementia risk: the Canadian study of health and aging. Neurology. 2008;70:9-16

43. Kolsch $\mathrm{H}$, Jessen F, Freymann $\mathrm{N}$, et al. ACE I/D polymorphism is a risk factor of alzheimer's disease but not of vascular dementia. Neurosci Lett. 2005:377:37-9.

44. Mortimer JA, Snowdon DA, Markesbery WR. The effect of APOE-epsilon 4 on dementia is mediated by alzheimer neuropathology. Alzheimer Dis Assoc Disord. 2009;23:152-7.

45. Qian L, Ding L, Cheng L, et al. Early biomarkers for post-stroke cognitive impairment. J Neurol. 2012;259:2111-8.

46. Rippon GA, Tang MX, Lee JH, Lantigua R, Medrano M, Mayeux R. Familial alzheimer disease in Latinos: interaction between APOE, stroke, and estrogen replacement. Neurology. 2006:66:35-40.

47. Treves TA, Bornstein NM, Chapman J, Klimovitzki S, Verchovsky R, Asherov A, Veshchev 1O, Korczyn AD. APOE-epsilon 4 in patients with alzheimer disease and vascular dementia. Alzheimer Dis Assoc Disord. 1996;10(4):189-91. 
48. Bour AM, Rasquin SM, Baars L, et al. The effect of the APOE-epsilon 4 allele and ACE-I/D polymorphism on cognition during a two-year follow-up in first-ever stroke patients. Dement Geriatr Cogn Disord. 2010;29:534-42.

49. Baum $L$, Chen $X$, Cheung WS, et al. Polymorphisms and vascular cognitive impairment after ischemic stroke. J Geriatr Psychiatry Neurol. 2007;20:93-9.

50. Arpa A, del Ser T, Goda G, Barba R, Bornstein B. Apolipoprotein E, angiotensin-converting enzyme and alpha-1-antichymotrypsin genotypes are not associated with post-stroke dementia. J Neurol Sci. 2003;210:77-82.

51. Slooter AJ, Tang MX, van Duijn CM, et al. Apolipoprotein E epsilon4 and the risk of dementia with stroke: a population-based investigation. JAMA. 1997;277:818-21.

52. Chapman J, Wang N, Treves TA, Korczyn AD, Bornstein NM. ACE, MTHFR, factor V Leiden, and APOE polymorphisms in patients with vascular and alzheimer's dementia. Stroke. 1998;29(7):1401-4.

53. Schrijvers EM, Schurmann B, Koudstaal PJ, et al. Genome-wide association study of vascular dementia. Stroke. 2012;43:315-9.

54. Morris CM, Ballard CG, Allan L, et al. NOS3 gene rs1799983 polymorphism and incident dementia in elderly stroke survivors. Neurobiol Aging. 2011;32:554e1-e6.

55. Battistin L, Cagnin A. Vascular cognitive disorder. A biological and clinical overview. Neurochem Res. 2010;35:1933-8.

56. Adair JC, Charlie J, Dencoff JE, et al. Measurement of gelatinase B (MMP-9) in the cerebrospinal fluid of patients with vascular dementia and alzheimer disease. Stroke. 2004;35:e159-62.

57. Candelario-Jalil E, Thompson J, Taheri S, et al. Matrix metalloproteinases are associated with increased blood-brain barrier opening in vascular cognitive impairment. Stroke. 2011;42:1345-50.

58. Wallin A, Ohrfelt A, Bjerke M. Characteristic clinical presentation and CSF biomarker pattern in cerebral small vessel disease. J Neurol Sci. 2012;322:192-6.

59. Hankey GJ, Ford AH, Yi Q, et al. Effect of B vitamins and lowering homocysteine on cognitive impairment in patients with previous stroke or transient ischemic attack: a prespecified secondary analysis of a randomized, placebo-controlled trial and meta-analysis. Stroke. 2013;44:2232-9.

60. Jin R, Yang G, Li G. Inflammatory mechanisms in ischemic stroke: role of inflammatory cells. J Leukoc Biol. 2010;87(5):779-89.

61. Zhang $L$, Yang $L$. Anti-inflammatory effects of vinpocetine in atherosclerosis and ischemic stroke: a review of the literature. Molecules. 2014;20(1):335-47.

62. Rothenburg LS, Herrmann N, Swardfager W, et al. The relationship between inflammatory markers and post stroke cognitive impairment. J Geriatr Psychiatry Neurol. 2010;23:199-205.

63. Narasimhalu K, Lee J, Leong $Y L$, et al. Inflammatory markers and their association with post stroke cognitive decline. Int J Stroke. 2015;10:513-8.

64. Kliper E, Bashat DB, Bornstein NM, et al. Cognitive decline after stroke: relation to inflammatory biomarkers and hippocampal volume. Stroke. 2013:44:1433-5.

65. Dong $H$, Li J, Huang L, et al. Serum MicroRNA profiles serve as novel biomarkers for the diagnosis of alzheimer's disease. Dis Markers. 2015;2015:625659.

66. Mehrabian S, Raycheva M, Petrova N, Janyan A, Petrova M, Traykov L. Neuropsychological and neuroimaging markers in prediction of cognitive impairment after ischemic stroke: a prospective follow-up study. Neuropsychiatr Dis Treat. 2015;11:2711-9.

67. Wattjes MP, Henneman WJ, van der Flier WM, et al. Diagnostic imaging of patients in a memory clinic: comparison of MR imaging and 64-detector row CT. Radiology. 2009;253:174-83.

68. Wahlund LO, Barkhof F, Fazekas F, et al. A new rating scale for age-related white matter changes applicable to MRI and CT. Stroke. 2001;32:1318-22.

69. Wardlaw JM, Smith EE, Biessels GJ, et al. Neuroimaging standards for research into small vessel disease and its contribution to ageing and neurodegeneration. Lancet Neurol. 2013;12:822-38.

70. Grysiewicz R, Gorelick PB. Key neuroanatomical structures for post-stroke cognitive impairment. Curr Neurol Neurosci Rep. 2012;12(6):703-8.

71. Heiss WD, Kidwell CS. Imaging for prediction of functional outcome and assessment of recovery in ischemic stroke. Stroke. 2014;45(4):1195-201.

72. Duering $M$, Righart $R$, Csanadi $E$, Jouvent $E$, Hervé $D$, Chabriat $H$, Dichgans M. Incident subcortical infarcts induce focal thinning in connected cortical regions. Neurology. 2012;79(20):2025-8.

73. Grau-Olivares M, Arboix A, Junqué C, Arenaza-Urquijo EM, Rovira M, Bartrés-Faz D. Progressive gray matter atrophy in lacunar patients with vascular mild cognitive impairment. Cerebrovasc Dis. 2010;30(2):157-66.
74. Kliper E, Ben Assayag E, Tarrasch R, Artzi M, Korczyn AD, Shenhar-Tsarfaty S, Aizenstein O, Hallevi H, Mike A, Shopin L, Bornstein NM, Ben BD. Cognitive state following stroke: the predominant role of preexisting white matter lesions. PLoS One. 2014;9(8):e105461.

75. Kooistra M, Geerlings MI, van der Graaf $Y$, et al. Vascular brain lesions, brain atrophy, and cognitive decline. The second manifestations of ARTerial disease - magnetic resonance (SMART-MR) study. Neurobiol Aging. 2014;35:35-41.

76. Kliper E, Ben Assayag E, Korczyn AD, Auriel E, Shopin L, Hallevi H, Shenhar-Tsarfaty S, Mike A, Artzi M, Klovatch I, Bornstein NM, Ben BD. Cognitive state following mild stroke: a matter of hippocampal mean diffusivity. Hippocampus. 2016;26(2):161-9. doi:10.1002/hipo.22500.

77. Heiss W-D, Zimmermann-Meinzingen S. PET imaging in the differential diagnosis of vascular dementia. J Neurol Sci. 2012;322:268-73.

78. Snowdon DA, Greiner LH, Mortimer JA, Riley KP, Greiner PA, Markesbery WR. Brain infarction and the clinical expression of alzheimer disease. JAMA. 1997;277:813-7.

79. Leys D, Henon H, Mackowiak-Cordoliani MA, Pasquier F. Poststroke dementia. Lancet Neurol. 2005:4:752-9.

80. Cechetto DF, Hachinski V, Whitehead SN. Vascular risk factors and alzheimer's disease. Expert Rev Neurother. 2008;8:743-50.

81. Pendlebury ST. Dementia in patients hospitalized with stroke: rates, time course, and clinico-pathologic factors. Int J Stroke. 2012;7(7):570-81.

82. Douiri A, McKevitt C, Emmett ES, Rudd AG, Wolfe CD. Long-term effects of secondary prevention on cognitive function in stroke patients. Circulation. 2013;128(12):1341-8.

83. Woods B, Aguirre E, Spector AE, Orrell M. Cognitive stimulation to improve cognitive functioning in people with dementia. Cochrane Database Syst Rev. 2012;2:CD005562.

84. Thiel A, Cechetto DF, Heiss WD, Hachinski V, Whitehead SN. Amyloid burden, neuroinflammation, and links to cognitive decline after ischemic stroke. Stroke. 2014;45:2825-9.

85. Arboix A, Blanco-Rojas L, Martí-Vilalta JL. Advancements in understanding the mechanisms of symptomatic lacunar ischemic stroke: translation of knowledge to prevention strategies. Expert Rev Neurother. 2014;14(3):261-76

86. Zanchetti A, Liu L, Mancia G, Parati G, Grassi G, Stramba-Badiale M, et al. Blood pressure and LDL-cholesterol targets for prevention of recurrent strokes and cognitive decline in the hypertensive patient: design of the European society of hypertension-Chinese hypertension league stroke in hypertension optimal treatment randomized trial. J Hypertension. 2014;32(9):1888-97.

87. PROGRESS Collaborative Group. Randomised trial of a perindopril-based blood-pressure-lowering regimen among 6,105 individuals with previous stroke or transient ischaemic attack. Lancet. 2001;358(9287):1033-41. Erratum in: Lancet. 2001;358(9292):1556. Lancet. 2002;359(9323):2120.

88. Diener HC, Sacco RL, Yusuf S, Cotton D, Ounpuu S, Lawton WA, et al. Effects of aspirin plus extended-release dipyridamole versus clopidogrel and telmisartan on disability and cognitive function after recurrent stroke in patients with ischaemic stroke in the prevention regimen for effectively avoiding second strokes (PRoFESS) trial: a double-blind, active and placebo-controlled study. Lancet Neurol. 2008;7(10):875-84.

89. Pearce LA, McClure LA, Anderson DC, Jacova C, Sharma M, Hart RG, et al. Effects of long-term blood pressure lowering and dual antiplatelet treatment on cognitive function in patients with recent lacunar stroke: a secondary analysis from the SPS3 randomised trial. Lancet Neurol. 2014;13(12):1177-85.

90. Ihle-Hansen H, Thommessen B, Fagerland MW, Øksengård AR, Wyller TB, Engedal K, Fure B. Blood pressure control to prevent decline in cognition after stroke. Vasc Health Risk Manag. 2015;11:311-6. doi:10.2147/VHRM.S82839.

91. Hornslien AG, Sandset EC, Bath PM, Wyller TB, Berge E. Effects of candesartan in acute stroke on cognitive function and quality of life: results from the Scandinavian candesartan acute stroke trial. Stroke. 2013;44(7):2022-4.

92. Manktelow BN, Potter JF. Interventions in the management of serum I ipids for preventing stroke recurrence. Cochrane Database Syst Rev. 2009;3:CD002091.

93. Collins R, Armitage J, Parish S, Sleight P, Peto R. Effects of cholesterol-lowering with simvastatin on stroke and other major vascular events in 20536 people with cerebrovascular disease or other high-risk conditions. Lancet. 2004;363(9411):757-67 
94. O'Brien EC, Greiner MA, Xian Y, Fonarow GC, Olson DM, Schwamm LH, et al. Clinical effectiveness of statin therapy after ischemic stroke: primary results from the statin therapeutic area of the patient-centered research into outcomes stroke patients prefer and effectiveness research (PROSPER) study. Circulation. 2015;132(15):1404-13.

95. Bath PM, Woodhouse L, Scutt P, Krishnan K, Wardlaw JM, Bereczki D, et al. Efficacy of nitric oxide, with or without continuing antihypertensive treatment, for management of high blood pressure in acute stroke (ENOS): a partial-factorial randomised controlled trial. Lancet. 2015;385(9968):617-28.

96. Kavirajan H, Schneider LS. Efficacy and adverse effects of cholinesterase inhibitors and memantine in vascular dementia: a meta-analysis of randomised controlled trials. Lancet Neurol. 2007;6(9):782-92.

97. Ballard C, Sauter M, Scheltens P, He Y, Barkhof F, van Straaten EC, et al Efficacy, safety and tolerability of rivastigmine capsules in patients with probable vascular dementia: the VantagE study. Curr Med Res Opin. 2008;24(9):2561-74.

98. Muresanu DF, Heiss WD, Hoemberg V, Bajenaru O, Popescu CD, Vester JC, et al. Cerebrolysin and recovery after stroke (CARS): a randomized, placebo-controlled, double-blind, multicenter trial. Stroke. 2016:47(1):151-9.

99. Mead GE, Hsieh CF, Hackett M. Selective serotonin reuptake inhibitors for stroke recovery. JAMA. 2013:310(10):1066-7.

100. Mead G, Hackett M, Lundstrom E, Murray V, Hankey G, Dennis M. The FOCUS, AFFINITY and EFFECTS trials studying the effect(s) of fluoxetine in patients with a recent stroke: a study protocol for three multicentre randomised controlled trials. Trials. 2015;16:369.

101. Alvarez-Sabin J, Roman GC. The role of citicoline in neuroprotection and neurorepair in ischemic stroke. Brain Sci. 2013;3(3):1395-414.

102. Wang X, Li J, Qian L, Zang XF, Zhang SY, Wang XY, Jin JL, Zhu XL, Zhang $X B$, Wang $Z Y, X u Y$. Icariin promotes histone acetylation and attenuates post-stroke cognitive impairment in the central cholinergic circuits of mice. Neuroscience. 2013;236:281-8. doi:10.1016/j.neuroscience.2012.12.074.

103. Lennon O, Galvin R, Smith K, Doody C, Blake C. Lifestyle interventions for secondary disease prevention in stroke and transient ischaemic attack: a systematic review. Eur J Prev Cardiol. 2014;21(8):1026-39.

104. Sun JH, Tan L, Yu JT. Post-stroke cognitive impairment: epidemiology, mechanisms and management. Ann Transl Med. 2014;2(8):80

105. Ihle-Hansen $H$, Thommessen B, Fagerland MW, Oksengard AR, Wyller TB, Engedal $\mathrm{K}$, et al. Multifactorial vascular risk factor intervention to prevent cognitive impairment after stroke and TIA: a 12-month randomized controlled trial. Int J Stroke. 2014;9(7):932-8.

106. Ihle-Hansen $H$, Thommessen B, Fagerland MW, Oksengard AR, Wyller TB, Engedal $\mathrm{K}$, et al. Effect on anxiety and depression of a multifactorial risk factor intervention program after stroke and TIA: a randomized controlled trial. Aging Ment Health. 2014;18(5):540-6.

107. Matz K, Teuschl Y, Firlinger B, Dachenhausen A, Keindl M, Seyfang L, et al. Multidomain lifestyle interventions for the prevention of cognitive decline after ischemic stroke: randomized trial. Stroke. 2015;46(10):2874-80.

108. Cumming TB, Tyedin K, Churilov L, Morris ME, Bernhardt J. The effect of physical activity on cognitive function after stroke: a systematic review. Int Psychogeriatr. 2012;24(4):557-67.

109. Stanek KM, Gunstad J, Spitznagel MB, Waechter D, Hughes JW, Luyster F, et al. Improvements in cognitive function following cardiac rehabilitation for older adults with cardiovascular disease. Int J Neurosci. 2011;121(2):86-93.

110. Marzolini S, Oh P, Mcllroy W, Brooks D. The effects of an aerobic and resistance exercise training program on cognition following stroke. Neurorehabil Neural Repair. 2013;27(5):392-402.

111. Moore SA, Hallsworth K, Jakovljevic DG, Blamire AM, He J, Ford GA, et al. Effects of community exercise therapy on metabolic, brain, physical, and cognitive function following stroke: a randomized controlled pilot trial. Neurorehabil Neural Repair. 2015;29(7):623-35.

112. Boss HM, Van Schaik SM, Deijle IA, de Melker EC, van den Berg BT, Scherder EJ, et al. A randomised controlled trial of aerobic exercise after transient ischaemic attack or minor stroke to prevent cognitive decline: the MovelT study protocol. BMJ Open. 2014;4(12):e007065.

113. Cicerone KD, Langenbahn DM, Braden C, Malec JF, Kalmar K, Fraas M, et al. Evidence-based cognitive rehabilitation: updated review of the literature from 2003 through 2008. Arch Phys Med Rehabil. 2011;92(4):519-30

114. Nair RD, Lincoln NB. Cognitive rehabilitation for memory deficits following stroke. Cochrane Database Syst Rev. 2007;3:CD002293.
115. Chung CS, Pollock A, Campbell T, Durward BR, Hagen S. Cognitive rehabilitation for executive dysfunction in adults with stroke or other adult non-progressive acquired brain damage. Cochrane Database Syst Rev. 2013:4:CD008391.

116. Cha YJ, Kim H. Effect of computer-based cognitive rehabilitation (CBCR) for people with stroke: a systematic review and meta-analysis. NeuroRehabilitation. 2013;32(2):359-68.

117. Nitsche MA, Paulus W. Excitability changes induced in the human motor cortex by weak transcranial direct current stimulation. J Physiol. 2000;527(Pt 3):633-9.

118. Siebner HR, Rothwell J. Transcranial magnetic stimulation: new insights into representational cortical plasticity. Exp Brain Res. 2003;148:1-16.

119. Chang WH, Bang OY, Shin Yl, Lee A, Pascual-Leone A, Kim YH. BDNF polymorphism and differential rTMS effects on motor recovery of stroke patients. Brain Stimul. 2014;7:553-8

120. Korczyn AD, Brainin M, Guekht A. Neuroprotection in ischemic stroke: what does the future hold? Expert Rev Neurother. 2015;15(3):227-9.

121. Elmlinger MW, Kriebel M, Ziegler D. Neuroprotective and anti-oxidative effects of the hemodialysate actovegin on primary rat neurons in vitro. Neuromolecular Med. 2011;13(4):266-74.

122. Mikhălova NM, Selezneva ND, Kalyn laB, Roshchina IF, Gavrilova SI. [Efficacy of actovegin in the treatment of elderly patients with vascular mild cognitive impairment.] Zh Nevrol Psikhiatr Im S S Korsakova. 2013;113(7 Pt 2):69-76. [In Russian]

123. Guekht A, Skoog I, Korczyn AD, Zakharov V, Eeg M, Vigonius U. A randomised double-blind, placebo-controlled trial of actovegin in patients with post-stroke cognitive impairment: ARTEMIDA study design. Dement Geriatr Cogn Dis Extra. 2013;3(1):459-67.

124. Skoog I, Korczyn AD, Guekht A. Neuroprotection in vascular dementia: a future path. J Neurol Sci. 2012;322(1-2):232-6.

\section{Submit your next manuscript to BioMed Central and we will help you at every step:}

- We accept pre-submission inquiries

- Our selector tool helps you to find the most relevant journal

- We provide round the clock customer support

- Convenient online submission

- Thorough peer review

- Inclusion in PubMed and all major indexing services

- Maximum visibility for your research

Submit your manuscript at www.biomedcentral.com/submit
) Biomed Central 